\title{
II. Tatsachenfeststellung als Voraussetzung eines effektiven Menschenrechtsschutzes
}

\section{Inhaltsverzeichnis}

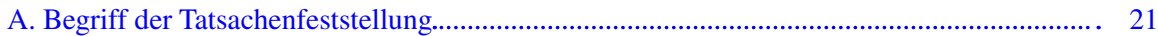

B. Funktion der Tatsachenfeststellung unter der EMRK ………………................................. 26

C. Rechtsgrundlagen des Beweisrechts der EMRK................................................................ 28

1. Beweisrecht in mitgliedstaatlichen Verfahren..................................................................... 28

2. Beweisrecht für das Verfahren vor dem EGMR................................................................... 29

\section{A. Begriff der Tatsachenfeststellung}

Die Anwendung jeder Rechtsnorm setzt die Verwirklichung ihrer Tatbestandsmerkmale durch einen bestimmten Sachverhalt voraus. ${ }^{1}$

Grundlage der Rechtsanwendung ist mit anderen Worten die Feststellung derjenigen Tatsachen, die für die rechtliche Beurteilung des fraglichen Sachverhalts erheblich sind. ${ }^{2}$ Der Begriff der Tatsachenfeststellung umschreibt diese beweismittelgestützte Rekonstruktion beziehungsweise Konstruktion von realen Geschehnissen ${ }^{3}$ zum Zwecke ihrer rechtlichen Einordnung und als Anknüpfungspunkt für die Fest-

\footnotetext{
${ }^{1}$ Zippelius, S. 91.

${ }^{2}$ Siehe auch Aemisegger/Robert, S. 1223.

${ }^{3}$ Nur reale Geschehnisse sind im Prinzip beweisbar, da nicht bewiesen werden kann, was nie geschehen, passiert, vorgefallen ist; vgl. Kaufmann, Beweisführung und Beweiswürdigung, S. 5. Zu eng Winkler, S. 41 f., welcher lediglich menschliche Verhaltensweisen im Hinblick auf einem bestimmten rechtlich gefassten Tatbestand dem Sachverhalt zuweist. Bestandteil des rechtserheblichen Sachverhalts können auch Naturkatastrophen sein, welche z. B. unter Art. 15 EMRK einen Grund für die Derogation der Pflichten aus der EMRK darstellen.
} 
legung von Rechtsfolgen durch ein dafür zuständiges Organ. ${ }^{4}$ Was hingegen Inhalt des Rechts ist, ist nur der Argumentation und keinem Beweis zugänglich. ${ }^{5}$ Verschiedene verfahrensrechtliche Regelungen knüpfen an die Unterscheidung zwischen Tatsache und Rechtsnorm an; so ist es klassischerweise Aufgabe des Gerichts, den Inhalt des Rechts von sich aus zur Geltung zu bringen (,iura novit curia“) - in vielen Verfahren hingegen Aufgabe der Parteien, den von ihnen behaupteten Sachverhalt zu beweisen.

In der eben vorgenommenen summarischen Beschreibung des Begriffs der Tatsachenfeststellung ist angedeutet, dass deren Zweck in erster Linie darin besteht, durch Beweismittel die effektiven Begebenheiten im Sinne einer objektiven (oder „materiellen“) ${ }^{6}$ Wahrheit zu rekonstruieren. Würde nämlich ein falscher Sachverhalt unter eine Rechtsnorm subsumiert, führte dies zu Rechtswidrigkeit und Unrecht. ${ }^{7}$ Insoweit hat das Beweisrecht eine der Durchsetzung des materiellen Rechts dienende Funktion.

Der Anspruch, mittels gerichtlicher Tatsachenfeststellung die objektive Wahrheit zu rekonstruieren, ${ }^{8}$ ist bei Lichte besehen jedoch nur eingeschränkt erfüllbar. Üblicherweise werden im Rahmen der Tatsachenfeststellung zwar Geschehnisse rekonstruiert, die sich in der Vergangenheit abgespielt haben, so dass es intuitiv möglich sein müsste, sich die Wahrheit durch Beweismittel zu erschließen. Nicht selten liegt das Problem jedoch bei den Beweismitteln: Oft muss zur Tatsachenfeststellung auf Aussagen von Personen zurückgegriffen werden, welche die in Frage stehenden Ereignisse als Beteiligte oder als Zeugen wahrgenommen haben. Solche Aussagen brauchen aber nicht die tatsächlichen Geschehnisse wiederzugeben. ${ }^{9}$ Ganz abgesehen davon, dass eine Aussage in einem Gerichtsverfahren auch von gewissen Interessen abhängen kann, ${ }^{10}$ variiert nämlich die Fähigkeit, visuelle, auditive und sensorische Reize ins Kurzzeitgedächtnis aufzunehmen und (richtig) einzuordnen situativ und je nach dem Vorwissen der wahrnehmenden Person erheblich. ${ }^{11}$ Vor allem aber unterliegen Erinnerungen mit Zeitablauf starker Verzerrung. ${ }^{12}$ Der EGMR hat in diesem Zusammenhang festgehalten, dass die Fähigkeit einer Zeugin, Geschehnisse in allen

\footnotetext{
${ }^{4}$ Bothe, Rn. 1.

${ }^{5}$ Vgl. zu der im schweizerischen Recht (prozessrechtlich) bedeutsamen Abgrenzung von Rechtsund Tatsachenfragen auch Aemisegger/Robert, S. 1223 ff. und Rhinow/Koller/Kiss/Thurnherr/ Brühl-Moser, Rn. 1113.

${ }^{6}$ Vgl. Geiser, S. 289.

${ }^{7}$ Winkler, S. 42.

${ }^{8}$ Vgl. für die EMRK Schorm-Bernschütz, S. 33.

${ }^{9}$ Ausführlich dazu Kaufmann, Beweisführung und Beweiswürdigung, S. 76. Zippelius, S. 91 f., spricht davon, dass die einzelne Wahrnehmung erst dann eine Erfahrungseinsicht darstelle, wenn sie mit anderen Erfahrungsgegebenheiten in widerspruchsfreiem Zusammenhang stehe.

${ }^{10}$ Eingehend dazu Ludewig/Baumer/Tavor, S. 17 ff.

${ }^{11} \mathrm{Vgl}$. Ludewig/Baumer/Tavor, S. 28 ff. sowie Kaufmann, Beweisführung und Beweiswürdigung, S. 76 ff., welcher anführt, dass neben dem Vorwissen auch z. B. Stress, Betroffenheit, Aufmerksamkeit und der Konsum von Betäubungsmittel sich auf die Fähigkeit auswirken können, Wahrnehmungen ins Kurzzeitgedächtnis aufzunehmen.

${ }^{12}$ Kaufmann, Beweisführung und Beweiswürdigung, S. 80 ff.
} 
Einzelheiten und mit Genauigkeit wiederzugeben, mit der Zeit abnehme ${ }^{13}$ und davon auch die Beweiskraft ihrer Aussage abhängig gemacht. ${ }^{14}$

Hier ist nicht der Platz, im Einzelnen auf die Verzerrungseffekte einzugehen, denen die menschliche Erinnerung ausgesetzt ist. ${ }^{15}$ Entscheidend ist vielmehr die Erkenntnis, dass Tatsachenfeststellung kaum je den Anspruch erfüllen wird, vergangene Geschehnisse genauso zu rekonstruieren, wie sie sich zugetragen haben. Selbst in den seltenen Fällen, in denen ein Sachverhalt durch Filmaufnahmen oder andere Beweismittel gewissermaßen ,umfassend“ dokumentiert ist, können vergangene Geschehnisse nur mehr oder weniger „objektiv“ rekonstruiert werden. Auch dann ist das rechtlich zu erfassende äußere Geschehen nämlich zumindest insofern interpretationsbedürftig, als es auf nicht messbaren inneren Wahrnehmungen und Beweggründen der handelnden Personen beruht, an deren Bestehen Rechtsnormen oft anknüpfen.

Noch größere Unsicherheiten bestehen, wenn sich die gerichtliche Tatsachenfeststellung auf einen zukünftigen Geschehensablauf bezieht. Der EGMR und nationalstaatliche Gerichte haben in diesem Zusammenhang regelmäßig die Frage zu beurteilen, ob einer Person bei einem Refoulement in einen anderen Staat das ernsthafte Risiko einer unmenschlichen Behandlung im Sinne von Art. 3 EMRK drohen würde. ${ }^{16}$ Erforderlich ist mithin eine Prognoseentscheidung, ${ }^{17}$ welche ausgehend von bestimmten Erfahrungswerten der Vergangenheit und Gegenwart eine wahrscheinlichkeitsbasierte Annahme zukünftiger Entwicklungen trifft. ${ }^{18}$ Dabei ist offensichtlich, dass keine Kongruenz des der Entscheidung zugrunde gelegten erwarteten Geschehensablaufs mit den zeitlich erst folgenden objektiven Tatsachen bestehen muss. In diesem Sinne darf von einer Beschwerdeführerin nicht verlangt werden, dass sie unwiderlegbare Beweise für die ihr im Falle einer Ausschaffung drohende unmenschliche Behandlung vorlegt, denn dies würde bedeuten, ,à lui demander de prouver l'existence d'un évènement futur, ce qui est impossible [...]. ${ }^{\text {“19,20 }}$

\footnotetext{
${ }^{13}$ Vgl. EGMR, Urteil vom 13. Juni 2017, Daşlik v. Türkei, § 57.

${ }^{14}$ Vgl. EGMR, Urteil vom 17. Februar 2004, Ipek v. Turkey, § 116.

${ }^{15}$ Mit einem umfassenden Überblick z. B. Heaton-Armstrong/Shepherd/Gudjonsson/Wolchover.

${ }^{16}$ Vgl. hierzu Beriger/Brunner, S. $195 \mathrm{ff}$.

${ }^{17}$ Vgl. zu diesem Begriff Ossenbühl, S. 731 ff. Der Begriff wird auch spezifisch für die Prüfung einer asylrechtsrelevanten „,begründeten Furcht vor zukünftiger Verfolgung“ verwendet, vgl. Frei, S. 55.

${ }^{18} \mathrm{Vgl}$. EGMR (Große Kammer), Urteil vom 23. August 2016, J.K. und andere v. Schweden, Concurring Opinion O'Leary, Ziff. 2: ,[...] [asylum cases] require the Court to craft prognostic judgments concerning what will or will not happen in the future if the applicants are returned to their country of origin. [Hervorhebung durch den Verfasser]."

${ }^{19}$ Vgl. EGMR, Urteil vom 14. Februar 2017, Allanazarova v. Russland, § 103, m.w.H.

${ }^{20} \mathrm{Vgl}$. für weitere Hinweise zu den diesbezüglichen Beweisschwierigkeiten EGMR, Urteil vom 26. Januar 2016, $R$ v. Russland, § 59: , ,[... [ requesting an applicant to produce „,indisputable“ evidence of a risk of ill-treatment in a third country would be tantamount to asking him to prove the existence of a future event, which is impossible, and would place a clearly disproportionate burden on him. Any such allegation always concerns an eventuality, something which may or may not occur in the future. Consequently, such allegations cannot be proven in the same way as past events. [...]."
} 
Auch ist nicht ausgeschlossen, dass eine Person, für welche das ernsthafte Risiko einer unmenschlichen Behandlung im Zielland (aufgrund der vorliegenden Beweismittel womöglich zu Recht) verneint wurde, nach dem Vollzug der Ausschaffung Opfer staatlicher Misshandlung wird. Kürzlich ist ein Fall bekannt geworden, in dem ein Tamile auf Grundlage eines Urteils des Bundesverwaltungsgerichts aus der Schweiz nach Sri Lanka abgeschoben und dort von den Behörden anschließend in Haft genommen und gefoltert wurde. Der Fall wurde in der Folge vom Anwalt des ausgewiesenen Asylsuchenden vor den EGMR gezogen, ${ }^{21}$ der die Schweiz wegen einer materiellen Verletzung von Art. 3 EMRK verurteilt hat. ${ }^{22}$

Weil eine naturwissenschaftlich genaue Rekonstruktion beziehungsweise Konstruktion von Tatsachen in Verwaltungs- und Gerichtsverfahren also nicht möglich ist und zudem oft zwischen den Parteien umstritten ist, was sich zugetragen hat, ${ }^{23}$ braucht es Normen, die bestimmen, auf welchen Sachverhalt (oder auf welche ,formelle Wahrheit") ${ }^{24}$ ein Gericht unter den immer gegebenen Unsicherheitsbedingungen bei seiner Entscheidung abzustellen hat. Diese Regeln, welche für die Tatsachenfeststellung in Verwaltungs- und Gerichtsverfahren Anwendung finden, werden als Beweisrecht bezeichnet. ${ }^{25}$

Selbstverständlich ändert die naturbedingte Unsicherheit jeder Tatsachenfeststellung nichts daran, dass ein ,gerechtes“ Beweisrecht in erster Linie darauf abzielen muss, den Sachverhalt so festzustellen, wie er sich zugetragen hat. Primärer Zweck eines jeden Beweisrechts ist es deshalb, eine möglichst objektive Rekonstruktion der effektiven Gegebenheiten und Geschehnisse zu ermöglichen und das Risiko einer fehlerhaften Sachverhaltsfeststellung auf ein Minimum zu reduzieren. ${ }^{26}$ Rechtsanwendende Organe sollen in Kenntnis sämtlicher für ihre Entscheidung erheblichen Tatsachen entscheiden ${ }^{27}$ und die Verantwortlichkeiten für die Tatsachenfeststellung müssen entsprechend verteilt werden. Gegenstand des Beweisrechts ist deshalb zunächst die Verteilung der Verantwortlichkeiten für die Ermittlung der relevanten Tatsachen sowie die Zulässigkeit einzelner Beweismittel. ${ }^{28}$

In der klassischen beweisrechtlichen Terminologie wird hinsichtlich der Verantwortlichkeit für die Ermittlung der relevanten Tatsachen zwischen der Verhandlungsmaxime und der Untersuchungsmaxime unterschieden. In Verfahren unter der Verhandlungsmaxime sind allein die Parteien für Einbringung und Beweis des von

\footnotetext{
${ }^{21}$ Vgl. dazu Schmid. Nach Bekanntwerden dieses und eines weiteren ähnlichen Falls hat das Bundesverwaltungsgericht im Urteil E-3726/2013 vom 14. Februar 2014 die Beschwerde eines Asylbewerbers gegen einen Ausweisungsentscheid des Bundesamts für Migration gutgeheißen, mit welcher der Beschwerdeführer eine neue Prüfung des Sachverhalts verlangte. In der Folge hat es sämtliche Parallelfälle kassiert und an die Vorinstanz zurückgewiesen.

${ }^{22}$ Vgl. EGMR, Urteil vom 26. Januar 2017, X. v. Schweiz.

${ }^{23} \mathrm{Zu}$ verschiedenen philosophischen Konzepten der „Wahrheit“ Naqvi, S. 249 ff.

${ }^{24}$ Vgl. Geiser, S. 289.

${ }^{25}$ Benzing, S. 11.

${ }^{26} \mathrm{Vgl}$. Binder, S. $15 \mathrm{f}$.

${ }^{27}$ Hinsichtlich internationaler Gerichte Benzing, S. 11.

${ }^{28} \mathrm{Vgl}$. Brower, S. 147.
} 
der Richterin zu beurteilenden Sachverhalts verantwortlich. Die Verhandlungsmaxime beruht auf der Überlegung, dass die gegensätzlichen Interessen der Parteien am Prozessausgang sowie der Umstand, dass sie über den Sachverhalt am besten informiert sind, zu einer vollständigen Sachverhaltsdarstellung führen. ${ }^{29}$ In Verfahren unter der Untersuchungsmaxime trägt hingegen letztlich die Richterin die Verantwortung für Sachverhalt und Beweis; ${ }^{30}$ gemildert wird diese Form gelegentlich durch Mitwirkungspflichten der Parteien, deren Missachtung sich unter bestimmten Umständen auf das Beweisergebnis auswirken kann. ${ }^{31}$

Das Beweisrecht hat neben der damit angesprochenen „Wahrheitsermittlungsfunktion" noch weitere Funktionen, die je nach Verfahrensgegenstand zu verschiedenen Ausgestaltungen der maßgeblichen Regelungen führen können: Insbesondere hat das Beweisrecht die Folgen des immer möglichen Fehlentscheids gerecht zu verteilen und die Kalkulierbarkeit des Tatsachenfindungsprozesses zu gewährleisten. ${ }^{32}$ Entsprechend muss festgelegt sein, welches Wahrscheinlichkeitsmaß für den Beweis einer Tatsache vorausgesetzt wird (Beweismaß), wie (sich unter Umständen widersprechende) Beweise zu würdigen sind (Beweiswürdigung) und welche der Parteien die nachteiligen Folgen der Beweislosigkeit trägt (Beweislastregeln). Teil des Beweisrechts bildet schließlich die Frage, inwiefern eine mit der Prüfung einer Konventionsverletzung befasste Instanz an die Tatsachenfeststellung einer vorangehenden Behörde gebunden ist (Regeln zur Sachverhaltskognition) und ob sie neue Beweismittel berücksichtigen darf (sog. Novenrecht). Hierbei spielen - gerade in Abwesenheit von ausdrücklichen verfahrensrechtlichen Regelungen - auch die funktionelle Eignung der mit der Tatsachenfeststellung befassten Instanz und ihre institutionelle Einbettung eine zentrale Rolle.

Die in diesem Zusammenhang häufig anzutreffende eingeschränkte Tatsachenkognition von Instanzengerichten hat nicht zuletzt die Funktion, den (theoretisch endlos führbaren) Streit über nicht mit letzter Gewissheit beweisbare Tatsachen irgendwann beiseitezulegen. Dabei spielt aber auch die Überlegung eine Rolle, dass mit zeitlicher und persönlicher Distanz einer richterlichen Entscheidungsträgerin die Nähe zum Sachverhalt schwindet. Insbesondere auf dieser Überlegung basiert die Zurückhaltung von Instanzengerichten, den erstinstanzlich festgestellten Sachverhalt zu korrigieren, zumal dieser nicht selten auf der Befragung von Parteien und der Würdigung ihrer Aussagen beruht. Im schweizerischen Asylverfahren beispielsweise führt das Bundesverwaltungsgericht als Beschwerdeinstanz kaum je Anhörungen durch; weil ihm damit der unmittelbare Eindruck der Person fehlt, der für die Einschätzung der Glaubhaftigkeit von Aussagen maßgebend sein kann, sollte es nicht unnötig in die Würdigung der Aussagen durch die erste Instanz eingreifen. ${ }^{33}$

\footnotetext{
${ }^{29}$ Vgl. Gygi, S. 207.

${ }^{30}$ Vgl. Kiener/Rütsche/Kuhn, Rn. 669.

${ }^{31}$ Vgl. zum Ganzen statt vieler Kiener/Rütsche/Kuhn, Rn. 713 f.

${ }^{32}$ Kaufmann, Bewiesen?, S. 1199 mit Bezug auf Gerichtsverfahren. Die Ausführungen Kaufmanns lassen sich aber auch auf die Tatsachenfeststellung in außergerichtlichen Verfahren übertragen.

${ }^{33}$ Um einen solchen Eindruck herzustellen, wird teilweise vorgeschlagen, Befragungen durch die erste Instanz audiovisuell aufzuzeichnen; vgl. Sonderegger/Kneer, Rn. 35.
} 


\section{B. Funktion der Tatsachenfeststellung unter der EMRK}

In der Rechtsprechung des EGMR kommt unter den verschiedenen genannten Funktionen des Beweisrechts insbesondere die Wahrheitsermittlungsfunktion zur Sprache. Auf Grundlage der durch Art. 1 EMRK begründeten Pflicht der Mitgliedstaaten, allen ihrer Hoheitsgewalt unterstehenden Personen die Konventionsrechte effektiv zuzusichern, leitete der EGMR aus den verschiedenen Konventionsgarantien weitreichende Ermittlungspflichten $a b,{ }^{34}$ deren Verletzung unabhängig von einer materiellen Verletzung der Konventionsrechte ${ }^{35}$ eine Verurteilung durch den EGMR nach sich ziehen kann. Wie der EGMR im diesbezüglich wegweisenden Fall El-Masri v. Mazedonien festgehalten hat, vermittelt der prozedurale Gehalt von Art. 3 EMRK in Fällen schwerer Menschenrechtsverletzungen ein eigenständiges „Recht auf Wahrheit“. ${ }^{36}$

Dieses „Recht auf Wahrheit“" kann wohl nicht nur auf Fälle schwerer Menschenrechtsverletzungen beschränkt werden: Verfahrensrechtliche Verpflichtungen der Konventionsstaaten ergeben sich nämlich schon aus dem allgemeineren Art. 13 EMRK, welcher ebenfalls auf eine wirksame innerstaatliche Untersuchung behaupteter Konventionsverletzungen abzielt, ${ }^{37}$ jedoch nicht auf Fälle beschränkt ist, die unter Art. 3 EMRK fallen. Umfangmäßig sind die prozeduralen Anforderungen, die der EGMR aus Art. 3 EMRK ableitet, weitgehend deckungsgleich mit den Anforderungen aus Art. 13 EMRK ${ }^{38}$ Eine effektive, auf die Ermittlung der Wahrheit ausgerichtete Untersuchung glaubhaft vorgebrachter materieller Konventionsverletzungen ist damit so oder anders ein eigenständiger prozeduraler Bestandteil der EMRK. ${ }^{39}$ Dass der EGMR die Untersuchungspflichten im Fall El-Masri gegen Mazedonien als Ausfluss des „Rechts auf Wahrheit" gemäß Art. 3 EMRK bezeichnete, war damit eher eine terminologische denn eine inhaltliche Neuerung.

Die terminologische Neuerung war und ist jedoch nicht bedeutungslos. Sie unterstreicht nämlich die hervorragende Bedeutung, die einer effektiven Untersuchung behaupteter Menschenrechtsverletzungen und der Ermittlung der Wahrheit in solchen Verfahren zukommt. Befriedigt wird nämlich einerseits das

\footnotetext{
${ }^{34}$ Ausführlich dazu Altermann, S. $106 \mathrm{ff}$.

${ }^{35}$ Vgl. z. B. die Fälle EGMR, Urteil vom 10. Oktober 2017, Tarjani v. Ungarn; EGMR, Urteil vom 3. Oktober 2017, D.M.D v. Rumänien; EGMR, Urteil vom 14. September 2017, Matevosyan v. Armenien; EGMR, Urteil vom 13. Juni 2017, Kosteckas v. Litauen.

${ }^{36}$ EGMR (Große Kammer), Urteil vom 13. Dezember 2010, El-Masri v. Mazedonien, § 191; ausführlich zu den sich diesbezüglich aus dem Urteil ergebenden Folgen Fabbrini, S. 18 ff.

${ }^{37}$ Vgl. EGMR (Große Kammer), Urteil vom 13. Dezember 2010, El-Masri v. Mazedonien, Concurring Opinion Tulkens, Spielmann, Sicilianos und Keller, $§ 6$.

${ }^{38} \mathrm{Vgl}$. Altermann, S. $85 \mathrm{ff}$.

${ }^{39}$ Zur Frage, ob dieser unter der EMRK bestehende Anspruch Ausprägung eines allgemeineren, völkergewohnheitsrechtlichen Anspruchs auf Wahrheit ist, vgl. Fabbrini, S. 19, m.w.H., sowie Naqvi, S. 254 ff.
} 
Bedürfnis der Opfer von Konventionsverletzungen nach Aufklärung der Geschehnisse und institutioneller Anerkennung des Geschehenen ${ }^{40}$ - oftmals Grundlage, um den Prozess der Verarbeitung anzustoßen und mit der (traumatisierenden) Vergangenheit abzuschließen. ${ }^{41}$ Die institutionelle Feststellung und Anerkennung der Geschehnisse ist insofern eine Form der Wiedergutmachung, die aus individueller Sicht wichtiger sein kann als monetäre Kompensation oder Bestrafung der Verantwortlichen. Anderseits trägt die institutionelle Aufklärung des Geschehenen aus gesamtgesellschaftlicher Perspektive entscheidend dazu bei, das Vertrauen in die Rechtsstaatlichkeit aufrechtzuerhalten. ${ }^{42}$ Nur durch effektive Untersuchungen kann rechtliche Verantwortung für gravierende Menschenrechtsverletzungen zugewiesen und können Täterinnen bestraft werden. ${ }^{43}$ Von der Bestrafung der Verantwortlichen geht auch eine abschreckende Wirkung aus; durch die (straf-)rechtliche Aufarbeitung des Vorgefallenen soll in diesem Sinne verhindert werden, dass sich Ähnliches wiederholt. ${ }^{44}$ Gerade in Fällen strukturell bedingter Menschenrechtsverletzungen kann durch die justizielle Feststellung der Tatsachen schließlich die Grundlage für die politische und gesellschaftliche Aufarbeitung des Geschehenen und damit letztlich auch für Versöhnung gelegt werden. ${ }^{45}$

Eine Gerichtsminderheit im Fall El-Masri v. Mazedonien hat diese Überlegungen wie folgt auf den Punkt gebracht hat:

\begin{abstract}
„En réalité, la recherche de la vérité constitue la finalité objective de l'obligation de mener une enquête ainsi que la raison d'être des exigences quant à la qualité de celle-ci (transparence, diligence, indépendance, accès, divulgation des résultats et contrôle). Pour la société en général, la volonté de rechercher la vérité contribue à renforcer la confiance envers les institutions et donc l'Etat de droit. Pour les personnes concernées, la famille et les proches des victimes, l'établissement de la vérité sur les faits et la reconnaissance des violations graves des droits de l'homme et du droit humanitaire constituent une forme de réparation aussi importante - et parfois même plus importante - qu'une indemnisation [...]. “46
\end{abstract}

\footnotetext{
${ }^{40}$ Vgl. EGMR (Große Kammer), Urteil vom 13. Dezember 2010, El-Masri v. Mazedonien, § 192.

${ }^{41}$ Naqvi, S. 249.

${ }^{42}$ EGMR (Große Kammer), Urteil vom 13. Dezember 2010, El-Masri v. Mazedonien, § 192.

${ }^{43}$ Zum Stellenwert dieses Aspekts vgl. Guidelines on eradicating impunity for serious human rights violations, wo in der Präambel statuiert wird, ,impunity must be fought as a matter of justice for the victims, as a deterrent to prevent new violations, and to uphold the rule of law and public trust in the justice system, including where there is a legacy of serious human rights violations $[\ldots]^{\prime \prime}$.

${ }^{44} \mathrm{Vgl}$. Updated Set of Principles for the Protection and Promotion of Human Rights Through Action to Combat Impunity, Prinzipien 2 and 3.

${ }^{45}$ Naqvi, S. 249.

${ }^{46}$ Vgl. EGMR (Große Kammer), Urteil vom 13. Dezember 2010, El-Masri v. Mazedonien, Concurring Opinion Tulkens, Spielmann, Sicilianos und Keller, § 6.
} 


\section{Rechtsgrundlagen des Beweisrechts der EMRK}

Die Feststellung des Sachverhalts - sowohl vor mitgliedstaatlichen Gerichten ${ }^{47}$ als auch vor dem EGMR ${ }^{48}$ - unterliegt bestimmten „Operationsregeln“ ${ }^{49}$ welche durch die EMRK beziehungsweise - im Verfahren vor dem EGMR - durch die EGMRVerfO vorgegeben sind.

\section{Beweisrecht in mitgliedstaatlichen Verfahren}

Wie einleitend bereits erläutert, zielt die vorliegende Arbeit darauf ab, den Vorgang der Tatsachenfeststellung in den verfahrensrechtlichen Instanzenzug und die Aufgabenteilung zwischen mitgliedstaatlichen Gerichten und EGMR einzubetten. Nach dem subsidiaritätsrechtlichen Prinzip der Primärverantwortung obliegt es in erster Linie den Mitgliedstaaten, die Konventionsgarantien zu schützen. Entsprechend setzt die Individualbeschwerde an den EGMR die Erschöpfung des innerstaatlichen Instanzenzugs voraus (Art. 35 Ziff. 1 EMRK). Die Durchsetzung der Konventionsrechte erfolgt zu einem großen Teil vor innerstaatlichen Verwaltungsbehörden und Gerichten und nicht vor dem EGMR. Für die Tatsachenfeststellung in diesen Verfahren ist im Grundsatz das Beweisrecht der nationalen Verfahrensordnungen anwendbar. Soweit es um die Untersuchung behaupteter Konventionsverletzungen geht, haben diese nationalen Verfahrensordnungen freilich die Vorgaben zu beachten, die sich aus der EMRK ergeben. ${ }^{50}$

Solche Vorgaben folgen insbesondere aus Art. 13 EMRK, wonach jede Person, die in ihren in der EMRK anerkannten Rechten oder Freiheiten verletzt worden ist, das Recht hat, bei einer innerstaatlichen Instanz eine wirksame Beschwerde zu erheben. Weitere spezifische Anforderungen für bestimmte Verfahrensarten ergeben sich aus Art. 6 EMRK (Recht auf ein faires Verfahren in ,zivilrechtlichen“ Streitigkeiten) und Art. 5 Ziff. 4 EMRK (Gerichtliche Überprüfung über die Rechtmäßigkeit eines Freiheitsentzugs). Der EGMR hat schließlich Untersuchungspflichten aus verschiedenen materiellen Konventionsrechten in Verbindung mit Art. 1 EMRK abgeleitet.

Die verfahrensrechtlichen Verpflichtungen aus den materiellen Konventionsrechten sind für die Tatsachenfeststellung in den Mitgliedstaaten ebenso verbindlich wie Art. 13 EMRK. Die Verletzung von Art. 13 EMRK oder einer Untersuchungspflicht aus einem materiellen Konventionsrecht zieht im Falle eines Weiterzugs nach Straßburg die Verurteilung eines Mitgliedstaats durch den EGMR nach sich.

\footnotetext{
${ }^{47}$ Vgl. dazu nachfolgend, II. C. 1.

${ }^{48} \mathrm{Vgl}$. dazu nachfolgend, II. C. 2.

${ }^{49}$ Zippelius, S. 93.

${ }^{50}$ Aemisegger/Roberts, S. 1228; Lanter, S. 57.
} 
Weil die Tatsachenfeststellung vor dem EGMR wie aufgezeigt an die Tatsachenfeststellung der innerstaatlichen Instanzen anknüpft, läge es nahe, diese nationalen Verfahrensordnungen als Teil des maßgeblichen Beweisrechts anzusehen. In diesem Sinne wäre es zwar höchst interessant, das Beweisrecht der 47 Mitgliedstaaten der EMRK im Hinblick auf die Untersuchung behaupteter Konventionsverletzungen zu vergleichen. Dies würde jedoch den Rahmen einer Dissertation sprengen und wäre wohl nur durch Einbeziehung von Autorinnen und Autoren aus den verschiedenen betroffenen Rechtsordnungen zu bewältigen. ${ }^{51}$ Es kann hier deshalb nur darum gehen, die Vorgaben darzustellen, die sich aus der EMRK für die Tatsachenfeststellung vor innerstaatlichen Behörden und Gerichten ergeben. Immerhin soll am Beispiel der Schweiz aufgezeigt werden, inwiefern die hier bestehenden Verfahrensordnungen die Anforderungen der EMRK implementiert haben; aufgrund der Rechtsprechung des EGMR zu den Verfahrensrechtsordnungen anderer Staaten wird vereinzelt auch auf anschauliche Beispiele in anderen Staaten hingewiesen.

\section{Beweisrecht für das Verfahren vor dem EGMR}

Was das Beweisrecht im Verfahren vor dem EGMR selbst betrifft, so ist dieses in der EMRK als dem ,gerichtseinsetzenden Vertrag “52 nur rudimentär geregelt. Nach Art. 38 EMRK ,prüft der Gerichtshof die Rechtssache mit den Vertretern der Parteien und nimmt, falls erforderlich, Ermittlungen vor [...]". Diese Bestimmung weist dem EGMR die Aufgabe der Tatsachenfeststellung zu und verleiht ihm entsprechende Kompetenzen, ${ }^{53}$ macht gleichzeitig aber auch deutlich, dass Ermittlungen nur vorzunehmen sind, wo sie erforderlich sind, etwa weil die Tatsachen in den vorgelagerten Verfahren der Konventionsstaaten mangelhaft ermittelt worden sind. Im Übrigen gibt die EMRK aber keinerlei Auskunft über die Modalitäten der Tatsachenfeststellung durch den Gerichtshof. ${ }^{54}$

Weitere die Tatsachenfeststellung des EGMR betreffende Bestimmungen finden sich in der EGMR-VerfO. Die Befugnis des Gerichtshofs, sich selbst eine Verfahrensordnung zu geben, basiert auf Art. 25 Bst. d EMRK, wonach das Plenum des Gerichtshofs die Verfahrensordnung des Gerichtshofs beschließt. Angesichts der spärlichen beweisrechtlichen Weichenstellungen in der EMRK selbst besitzt der Gerichtshof damit große Freiheiten, was die Ausgestaltung des Beweisrechts in seinem eigenen Verfahren angeht. ${ }^{55}$

\footnotetext{
${ }^{51} \mathrm{Vgl}$. zu den methodologischen Schwierigkeiten einer solchen „,diffusen“ Untersuchung der Wirkungsweise der EMRK-Vorgaben in den Verfahrensrechtsordnungen der Mitgliedstaaten Christoffersen, S. 25.

${ }^{52}$ Benzing, S. 49.

${ }^{53}$ Schorm-Bernschütz, S. 47.

${ }^{54}$ Noch zum inhaltlich weitgehend deckungsgleichen vormaligen Art. 38 Ziff. 1 Bst. a EMRK, welcher seit 1 Juni 2010 nicht mehr in Kraft ist, Schorm-Bernschütz, S. 47.

${ }^{55}$ Vgl. Meyer-Ladewig, Rn. 6 zu Art. 25 EMRK.
} 
Die Verfahrensordnung des EGMR umfasst insbesondere beweisrechtliche Bestimmungen zum Eingabeschluss (Art. 38 EGMR-VerfO), zu den Mitwirkungsrechten und -pflichten der Parteien (Art. 44A-44E EGMR-VerfO), zu den möglichen Inhalten einer Staaten- und einer Individualbeschwerde (Art. 46-47 EGMR-VerfO) und zu den Hearings, denen auch eine Tatsachenermittlungsfunktion zukommen kann (Art. 63-70 EGMR-VerfO).

Weite Teile der oben skizzierten beweisrechtlichen Fragestellungen sind damit weder in der EMRK noch in der EGMR-VerfO geregelt. Insbesondere finden sich dort keine Anhaltspunkte zu Fragen der Beweiswürdigung und des Beweismaßes, zur Prüfungsdichte des EGMR in Sachverhaltsfragen und zum Novenrecht. Der EGMR hat bezüglich dieser Fragen in seiner Rechtsprechung in Form von wiederkehrenden Leitsätzen aber konsistente Standards entwickelt, auf welche sowohl Staaten als auch Beschwerdeführerinnen in Verfahren vor dem EGMR immer wieder Bezug nehmen. Diese Leitsätze können zwar nicht als die EMRK ergänzendes Völkergewohnheitsrecht angesehen werden, stellen aber doch verbindliche prozessuale Rechtsquellen dar und sind insofern für die vorliegende Analyse miteinzubeziehen. ${ }^{56}$ Eine wichtige Rolle insbesondere für die „Sachverhaltskognition“ und das „Novenrecht“" spielt der Subsidiaritätsgrundsatz, der das Verhältnis von nationalstaatlichen Gerichten zum EGMR ganz allgemein prägt. Im nachfolgenden Kapitel soll deshalb dessen Gehalt näher erläutert werden.

\footnotetext{
${ }^{56}$ Umfassend zum Richterrecht als eigenständiger Rechtsquelle des Völkerprozessrechts Benzing, S. 86 ff., zusammenfassend S. 98 f.
}

Open Access Dieses Kapitel wird unter der Creative Commons Namensnennung 4.0 International Lizenz (http://creativecommons.org/licenses/by/4.0/deed.de) veröffentlicht, welche die Nutzung, Vervielfältigung, Bearbeitung, Verbreitung und Wiedergabe in jeglichem Medium und Format erlaubt, sofern Sie den/die ursprünglichen Autor(en) und die Quelle ordnungsgemäß nennen, einen Link zur Creative Commons Lizenz beifügen und angeben, ob Änderungen vorgenommen wurden.

Die in diesem Kapitel enthaltenen Bilder und sonstiges Drittmaterial unterliegen ebenfalls der genannten Creative Commons Lizenz, sofern sich aus der Abbildungslegende nichts anderes ergibt. Sofern das betreffende Material nicht unter der genannten Creative Commons Lizenz steht und die betreffende Handlung nicht nach gesetzlichen Vorschriften erlaubt ist, ist für die oben aufgeführten Weiterverwendungen des Materials die Einwilligung des jeweiligen Rechteinhabers einzuholen. 